\title{
Surface Engineering of poly(methylmethacrylate): Effects on Fluorescence Immunoassay
}

Running title: Surface Engineering of poly(methylmethacrylate): Effects on Fluorescence Immunoassay

Running Authors: Akers et al.

\section{Peter W. Akers}

School of Chemical Sciences, University of Auckland, Private Bag 92019, Auckland 1142, New Zealand; and MacDiarmid Institute for Advanced Materials and Nanotechnology, PO Box 600, Wellington 6140, New Zealand

Nam Cao Hoai Le

Microfluidics Laboratory, CSIRO Manufacturing Flagship, Clayton South MDC, Victoria 3169, Australia

Andrew R. J. Nelson

Australian Centre for Neutron Scattering, Australian Nuclear Science and Technology Organisation, Locked Bag 2001, Kirrawee DC, NSW 2232, Australia

Milena McKenna

Biomedical Diagnostics Institute, Dublin City University, Glasnevin Avenue, Dublin 9, Ireland

Christy O'Mahony

School of Chemistry and Biochemistry, Georgia Institute of Technology, Atlanta, Georgia 303320400, United States

\section{Duncan J. McGillivray}

School of Chemical Sciences, University of Auckland, Private Bag 92019, Auckland 1142, New Zealand; and MacDiarmid Institute for Advanced Materials and Nanotechnology, PO Box 600, Wellington 6140, New Zealand

Vladimir Gubala $\left.{ }^{\ddagger a}\right)$

Medway School of Pharmacy, University of Kent, Chatham Maritime, Kent, ME4 4TB, United Kingdom

David E. Williams ${ }^{\ddagger}$

School of Chemical Sciences, University of Auckland, Private Bag 92019, Auckland 1142, New Zealand; and MacDiarmid Institute for Advanced Materials and Nanotechnology, PO Box 600, Wellington 6140, New Zealand

${ }^{a)}$ Corresponding author's electronic mail:V.Gubala@kent.ac.uk

"These authors contributed equally. 
We present surface engineering modifications through chemistry of poly(methylmethacrylate) - PMMA- that have dramatic effects on the result of surfacebound fluorescence immunoassays, both for specific and non-specific signals. We deduce the most important effect to be clustering of antibodies on the surface leading to significant self-quenching. Secondary effects are attributable to the formation of sparse multilayers of antibody. We compare PMMA as an antibody support surface with UV-ozone oxidised PMMA and also to substrates that were, after the oxidation, surface modified by a 4-unit poly(ethyleneglycol) carboxylic acid $\left(\mathrm{PEG}_{4}\right)$, a branched tri-carboxylic acid, and a series of carboxylic acid-terminated dendrimers, from generation 1.5 to 5.5. Fluorescence immunoassay and neutron reflectometry were used to compare the apparent antibody surface loading, antigen binding and non-specific binding on these various surfaces using anti-human IgG as a model antibody, chemically coupled to the surface by amide formation. Simple physical adsorption of the antibody on PMMA resulted in a thick antibody multilayer with small antigen binding capacity. On the carboxylated surfaces, with chemical coupling, a simple monolayer was formed. We deduce that antibody clustering was driven by conformational inflexibility and high carboxylate density. The $\mathrm{PEG}_{4}$-modified surface was the most conformationally flexible. The dendrimer-modified interfaces showed a collapse and densification. In fluorescence immunoassay, the optimal combination of high specific and low non-specific fluorescence signal was found for the G3.5 dendrimer 


\section{INTRODUCTION}

One of the most challenging issues in the wide field of new generation biosensors is the development of surfaces that retain the activity of immobilized biomolecules whilst minimizing non-specific binding $[1,2]$. For biomedical diagnostics applications, the specific binding activity of a surface-adsorbed antibody for its antigen and the rejection of non-specific binding are critical factors that govern the sensitivity, dynamic range, and reproducibility of the immunoassays. Some existing limitations of traditional immunoassays were recently attempted to be solved by introduction of biologicallymimicking nanomaterials $[3,4]$. The advances in miniaturized optical, acoustic and electric sensors introduced both new possibilities and challenges in the control of interactions of biomolecules with nanoscale biosensor surfaces [5]. The established strategies based on physical adsorption [6], covalent, and bioaffinity mechanisms of immobilization have been well-documented [7-12]. The motivation behind these methods is to ensure optimum antigen binding activity, interpreted as the most favourable orientation of the immobilized antibody, in which the antigen-binding site is oriented away from the surface itself [13]. The two most common methods of immobilizing antibodies to surfaces involve either noncovalent physical adsorption to a detection surface through non-specific hydrophobic interactions (physical adsorption) or non-specific, covalent immobilization (chemisorption), typically to amine reactive surfaces $[14,15]$. Although both methods result in randomly-oriented antibody molecules on the surface, with up to $90 \%$ antibody that is in an inactive orientation, these methods are commonly used because of their simplicity. A potential issue with these methods is that the non-active antibodies exposed to the solution could act to promote non-specific binding: any such effect would be dependent on the nature of the antibody, particularly on the solution-exposed amino acid 
composition of the adsorbed layer. Site-specific immobilization methods are also available [16-21], which do lead to significantly enhanced antigen binding efficiency, though at the expense of more complex procedures [16, 22, 23].

Antibody adsorption onto surfaces, and the subsequent binding of an antigen, is critically dependent on the nature of the surface, on the antibody and on the antigen. Antibody adsorption depends on the $\mathrm{pH}$ and salt concentration [24] (which determines the charge on the antibody relative to the surface charge), hydrophobicity or hydrophilicity of the surface (that is, the balance of Van der Waals and solvation interactions with hydrophobic and hydrophilic amino acids of the antibody), the surface loading and whether there is a specific surface binding chemistry employed: for example the use of amide coupling to a carboxylated surface from amines of the antibody selects for lysines, and so may bias the orientation dependent on the location of lysines on the antibody surface [25-29]. Antigen binding to a surface-adsorbed antibody depends on the availability of binding sites which is affected by the average orientation of the antibody. Antigen binding then further depends on steric hindrance and the geometry of the antigen epitope. These factors can be altered by surface loading and the interaction of the antibody with the surface [30]. Adsorption should also occur so as to maximise the interaction energy between the antibody and the surface, which in general would require maximising the interfacial contact area and hence should promote "flat-on" adsorption. The consequent effect of epitope geometry on antigen binding can be seen in comparisons of different antibodies. Thus, the human chorionic gonadotrophin antigen, $\mathrm{hCG}$, binds at essentially 1:1 molar ratio with its surface-adsorbed antibody at low coverage of the antibody, but other antigen antibody pairs show essentially zero binding [31]. As the antibody surface coverage increases then intermolecular 
interactions seem to result in an irregular packing, describable as an irregular stack of plates [32]. The antibody binding site may then become more accessible for binding - bound antigen/surface antibody may increase - or else steric hindrance may diminish the ratio of bound antigen/surface antibody.

The term "soft interface" can be used to describe surfaces that have some conformational flexibility and can in some sense reorganise in response to adsorption of other molecules. In the present paper, we set out to explore the utility of the concept of "conformational flexibility" or "softness" of a surface as a guide to the design of effective interfaces for antibody immobilisation, using anti-human IgG as a model. We compare the antigen binding of the antibody surface-bound to a number of different carboxylated surfaces. These surfaces, shown in figure 1, were derived from poly(methylmethacrylate) [33], PMMA, carboxylated by UV-ozone treatment and then chemically modified.

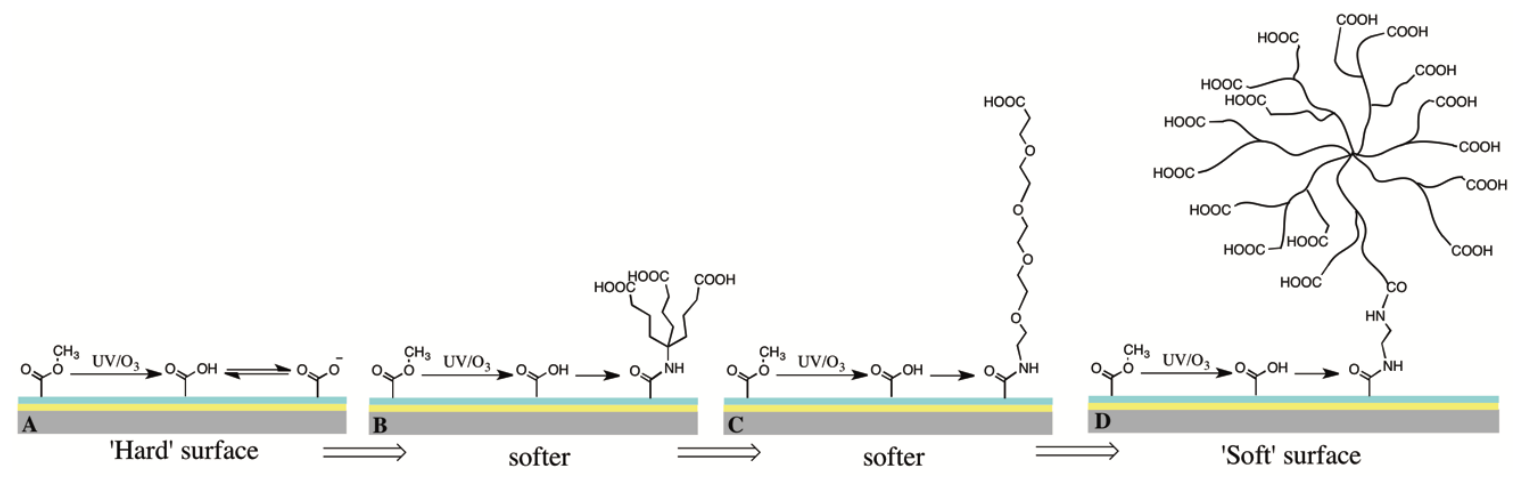

FIGURE 1. Illustration of the different surfaces prepared and their qualitative classification as 'hard' and 'soft'. To limit the number of variables, all surfaces were designed with the terminal $-\mathrm{COOH}$ group, which enabled the covalent attachment of antibodies via the available Lys groups (using $\varepsilon-\mathrm{NH}_{3}{ }^{+}$Lysine molecules). Four types of surfaces were studied: A - oxidized PMMA, B - aminotriacid dendron, purchased as 4-amino-4-[3-(1, 1dimethylethoxy) -3-oxopropyl] -1, 7-bis(1, 1-dimethylethyl) ester heptanedioic acid, which was hydrolysed to the tri-acid prior to surface attachment, $\mathrm{C}-$ amino- $\mathrm{PEG}_{4}-\mathrm{COOH}$ and $\mathrm{D}$ - half-generation PAMAM dendrimer modified surfaces. The a-priori anticipated "softness" increases from left to right. As noted later in the paper, the results did not entirely support this classification. 
By comparing results from two complementary techniques we show unexpected effects of surface composition on antibody immobilisation and on the resultant specific and non-specific binding of the protein antigen (IgG in this case). We first present results using fluorescently labelled reagents to measure the apparent amount of surface antibody, and the signal from both complementary and non-complementary antigens. We then compare these results with neutron reflection measurements, which give absolute amounts of material on the surface. These two techniques give very different results for the different surfaces. We infer that important effects on the specific and non-specific signals in fluorescent immunoassay are not due to variations in antibody loading or antigen binding capacity. Instead, we deduce that these effects derive from antibody clustering on the surface leading to self-quenching. We speculate that surface conformational flexibility and surface charge are important factors driving antibody clustering.

\section{EXPERIMENTAL}

\section{A. Materials}

Polymethylmethacrylate (PMMA) sheets $(0.25 \mathrm{~mm}$ thick, impact modified, average $\mathrm{MW}=120,000 \mathrm{Da}$ ) were supplied by Goodfellow Cambridge Limited (Huntingdon, England) and cut into small pieces. Gold-coated standard glass slides (Ti/Au $=2 \mathrm{~nm} / 30 \mathrm{~nm}, 26 \mathrm{~mm} \times 76 \mathrm{~mm}, 1 \mathrm{~mm}$ thick) were purchased from Phasis Sarl (Geneva, Switzerland). N-(3-dimethylaminopropyl)-N'-ethylcarbondiimide hydrochloride (EDC), ethylenediamine (EDA), N-Hydroxysuccinimide (NHS), 2-(N-morpholino)ethanesulfonic acid (MES), phosphate-buffered saline (PBS, $\mathrm{pH}$ 7.4) tablets, PAMAM dendrimer (generation 1.5, 2.4, 3.5, 4.5 and 5.5), and toluene were purchased from Sigma Aldrich 
(Dublin, Ireland). The anti-human IgG, human IgG and rabbit IgG were purchased from Molecular Probes ${ }^{\mathrm{TM}}$ (Eugene, OR), the NTproBNP was purchased from HyTest (Turku, Finland). For neutron reflectometry experiments, PMMA (average $\mathrm{M}_{\mathrm{w}} \sim 120,000 \mathrm{Da}$ ), IgG from human serum, anti-Human IgG (Fab specific) antibody produced in goat and G4.5 PAMAM dendrimer $(5 \% \mathrm{w} / \mathrm{w}$ solution in methanol) were obtained from Sigma Aldrich (NSW, Australia) and used without further purification. The amino-triester was purchased from Frontier Scientific (UT, United States: product NTN1963) and converted to the triacid before use in NR experiments. The Amino- $\mathrm{dPEG}^{\circledR}{ }_{4}$-acid (Aminoethyl-trioxyethylene acetic acid) was purchased from Quantabiodesign (Plain City, OH: product 10234)

\section{B. Experimental set up and Methodology}

\section{Preparation of PMMA thin film by spin coating}

The method followed that described by Le et al. [34]. PMMA sheets were cut into small pieces and dissolved in toluene at concentration ranging from 0.1 to $0.5 \%(\mathrm{w} / \mathrm{v})$ to make the raw PMMA solution. The solution was sonicated for 15 min to dissolve PMMA pieces. The PMMA solution was then filtered through a PTFE filter (pore size $0.2 \mu \mathrm{m}$ ) (Chromafil Xtra PTFE-20/25 Macherey-Nagel, Duren, Germany) to eliminate the precipitates and dust particles. $0.25 \%(\mathrm{w} / \mathrm{v})$ PMMA solution was then spin coated onto Aucoated glass slides or silicon wafers at $2000 \mathrm{rpm}$ for $45 \mathrm{~s}$. The acceleration and deceleration from $0 \mathrm{rpm}$ to the desired spin speed and from spin speed to $0 \mathrm{rpm}$ took place in $5 \mathrm{~s}$. The films were first cured at room temperature over-night and then cured in an oven for $1 \mathrm{~h}$ at $80^{\circ} \mathrm{C}$. These parameters resulted in very smooth and uniform PMMA films on both $\mathrm{Au}-$ coated glass slides and silicon wafers.

\section{Modification of PMMA surface}


$\mathrm{UV} / \mathrm{O}_{3}$ treatment was performed using a commercial ozone cleaning and activation system (PSD-UV, Novascan Technologies, Ames, IA, USA). The specifications indicated at $50 \mathrm{~W}$ power setting, approximately $50 \%$ of the total lamp output power is delivered around the $254 \mathrm{~nm}$ peak and $5 \%$ around the $185 \mathrm{~nm}$ peak. We found that $8 \mathrm{~min}$ of $\mathrm{UV} / \mathrm{O}_{3}$ treatment was optimal for the thin PMMA film, balancing activation of the surface against etching. For binding of the amine-terminated surface modifiers, the fresh $\mathrm{UV} / \mathrm{O}_{3}$ treated PMMA surface was allowed to react for 30 min with an MES (0.01 M, pH 4.5) solution containing $100 \mathrm{mM}$ EDC and $50 \mathrm{mM}$ NHS to form reactive NHS-esters. The substrates were rinsed 3 times with DI water and then immersed in a $50 \mathrm{mM}$ solution of either Amino-

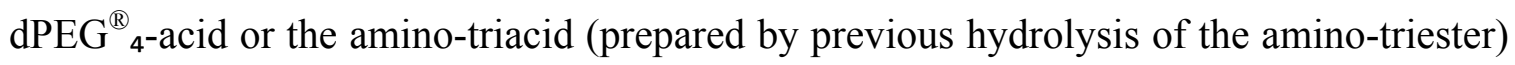
in PBS for 2 hours. The substrates were then thoroughly washed with PBS Tween solution and rinsed with DI water and finally dried in a $\mathrm{N}_{2}$ stream.

For surface modification with carboxylate-terminated dendrimers, a 2-stage procedure was used, involving coating the $\mathrm{UV} / \mathrm{O}_{3}$ treated PMMA surface with ethylene diamine (EDA) linkers upon which the dendrimer layer was built. Firstly, the fresh $\mathrm{UV} / \mathrm{O}_{3}$ treated PMMA surface was immersed in a solution containing $100 \mathrm{mM}$ EDC and 50mM EDA for 20 min. The substrates were then rinsed thoroughly with EtOH (3×), immersed in DI water for 10 min and dried in a $\mathrm{N}_{2}$ stream. An activated carboxyl-terminated dendrimer solution was prepared according to previously published protocols[35]. As an example, 1 $\mu$ mol of PAMAM G1.5 dendrimer (with 16-COOH surface groups) was mixed with 96 $\mu \mathrm{mol}$ of EDC (6.0 equiv. per one $-\mathrm{COOH}$ group), $24 \mu \mathrm{mol}$ of NHS, (1.5 equiv. per one $\mathrm{COOH}$ group), $16 \mu \mathrm{l}$ of $\mathrm{HCl}$ and topped up to final volume of $2 \mathrm{~mL}$ with $\mathrm{DI}$ water. The sample was allowed to shake for $20-25 \mathrm{~min}$ at $600 \mathrm{rpm}$ using vortex mixer. The EDA 
coated slide was then immersed in the respective dendrimer solution contained in a Petri dish and was allowed to react for $2 \mathrm{~h}$, washed extensively with PBS Tween solution, rinsed with DI water and finally dried in a $\mathrm{N}_{2}$ stream. The dendrimer coated slides were stored in a vacuum chamber until used. Water contact angle measurement and Atomic Force Microscopy (ESI, Figures S.1 and S.2) were used to check the progress and uniformity of the surface modification.

\section{Antibody adsorption and binding activity by fluorescence measurement}

The direct-binding fluorescence immunoassay was performed on standard-size microscope slides, spin-coated with PMMA and modified with the corresponding linker (e.g., tris-acid, $\mathrm{PEG}_{4}$, dendrimers) as described in details in the experimental section. The $-\mathrm{COOH}$ groups on such modified slides were then further activated with a solution containing $100 \mathrm{mM}$ EDC and $50 \mathrm{mM}$ NHS to form NHS-esters. The activated slide was then covered with a solution of goat anti-human IgG solution (50 $\mu \mathrm{g} / \mathrm{ml}$ in $0.01 \mathrm{M}$ PBS, $\mathrm{pH}$ 7.4) and allowed to react at $37^{\circ} \mathrm{C}$ for two hours. In order to minimize evaporation of the solvent from the slide surface, the slides were placed in a humidity and temperature controlled chamber. The surface was then washed with PBS containing Tween $20(0.2 \%$, $\mathrm{v} / \mathrm{v}$ ) and then with PBS. The slide was subsequently immersed in a PBS solution containing BSA $(1 \%, w / v)$ for one hour. The slide was finally thoroughly rinsed with PBS $(3 \times)$ and dried under a stream of $\mathrm{N}_{2}$. The goat anti-human IgG antibody-coated slides were used in the direct binding assay with Cy5-labeled human IgG. Note: In order to quantify the amount of goat anti-human IgG bound on the substrate surface, Cy5-labeled goat antihuman IgG was spotted on the NHS-ester activated surface. 
The specific binding of human IgG on the goat anti-human IgG antibody-coated surface was performed as follows. The goat anti-human IgG coated slide, dried under the stream of $\mathrm{N}_{2}$ was spotted in an array of 3 spots ( $3 \mu \mathrm{L}$ per spot) with a $5 \mu \mathrm{g} / \mathrm{mL}$ solution of Cy5-labeled human IgG (0.01 M PBS, pH 7.4). To study the non-specific binding on the antibody-coated surfaces, the slide was spotted in an array of 3 spots ( $3 \mu \mathrm{L}$ per spot) with a $5 \mu \mathrm{g} / \mathrm{mL}$ solution of Cy5-labeled NTproBNP (0.01 M PBS, $\mathrm{pH} 7.4)$. The spotted slide was immediately placed in a humidity chamber to minimize solvent evaporation at $37^{\circ} \mathrm{C}$ for two hours. The slide was then rinsed with a PBS solution containing Tween $20(0.2 \%$, $\mathrm{v} / \mathrm{v}), \operatorname{PBS}(2 \times)$ and dried under a stream of $\mathrm{N}_{2}$.

The fluorescence intensities of the spots were measured on PerkinElmer ScanArray Express (PerkinElmer, Massachusetts, USA) with laser excitation wavelength of $633 \mathrm{~nm}$, emission filter wavelength of $670 \mathrm{~nm}$, and analyzed using ImageJ software. The fluorescence images and the analyzed fluorescence data are summarized in figures S.3-S.5.

\section{Neutron reflectometry}

Neutron reflectometry was performed on the Platypus time-of-flight neutron reflectometer at ANSTO [36] using an incident beam spectrum with neutron wavelengths $2.8 \AA<1<18 \AA .24 \mathrm{~Hz}$ neutron pulses were generated using a disc chopper system, collimated, and reflected from the sample at $0.8^{\circ}, 2.5^{\circ}$ and $5.0^{\circ}\left(0.006<\mathrm{Q} / \AA^{-1}<0.391\right)$, before detection with a $2 \mathrm{D}$ position sensitive detector. The reflection of a sample is calculated as the ratio of the reflected and incident beam intensities. Corrections for instrumental resolution are taken into account during analysis and reflection is expressed as a function of $Q$ : 


$$
Q=\frac{4 \pi}{\lambda} \sin \theta
$$

where $\theta$ is the angle of incidence and $\lambda$ is the wavelength of the radiation. Modeling of the data involves an optical matrix method in which a model of the surface structure is created and divided into homogeneous layers. Each homogeneous layer is defined by a thickness, interfacial roughness, volume fraction of water and neutron scattering length density of the material and water. The neutron scattering length density (nSLD) of a molecule is the sum of all neutron scattering lengths (a measure of atomic interaction with neutrons which varies from isotope to isotope) divided by the molecular volume. Two measurements were made on each sample using different isotopic compositions of water (i.e., $\mathrm{D}_{2} \mathrm{O}$ and $\mathrm{H}_{2} \mathrm{O}$ ) as they have different $\mathrm{nSLD}$, to allow co-refinement of constrained models reducing model ambiguity and allowing calculation of the hydration of each of the layers. Model refinement and optimization involved least squares minimization using the Motofit [37] data analysis program. Error values were obtained using Monte Carlo error analysis methods [38]. Mass loadings on the surface were calculated from the derived layer thicknesses and volume fractions using the mass density of the various components. For the protein, the mass density was calculated from the molecular volume and molecular mass.

\section{RESULTS AND DISCUSSION}

\section{A. Fluorescence measurements show a very wide range of apparent surface coverage of immobilized antibody and of non-specific: specific binding ratio, with an optimum across the dendrimer series}


Figure 2A shows the fluorescence signal from the surface bound goat-anti human-IgG antibody, its specifically-bound antigen, human IgG, and the non-specific signal from binding of the antigen NT-proBNP. A wide range in surface-bound antibody signal is observed, over an order of magnitude, for the different surface treatments. Figure $2 \mathrm{~B}$ shows that the fluorescence signal for both specific and non-specific antigens correlates with that for the surface-bound antibody, though with some outliers for particular surface treatments that are explored later. Notable is the relatively high specific binding signal and low nonspecific binding signal observed for the G3.5 dendrimer as linker. Figure 2C shows the ratio of non-specific to specific binding signal, illustrating that this ratio is generally correlated with the fluorescence signal for the surface-bound antibody, apart from the oxidized PMMA surface which had the lowest fluorescence signal for surface-bound antibody, the lowest specific antibody signal and a ratio of non-specific to specific binding $>1$. Figure $2 \mathrm{C}$ again highlights that the G3.5 dendrimer linker is a low-side outlier and the G5.5 dendrimer a high-side outlier. The amount of surface-bound antibody, determined by neutron reflection, is also shown on figure $2 \mathrm{~A}$. It is immediately evident that the fluorescence and neutron results do not correlate. Neutron reflection results are developed in detail below. 


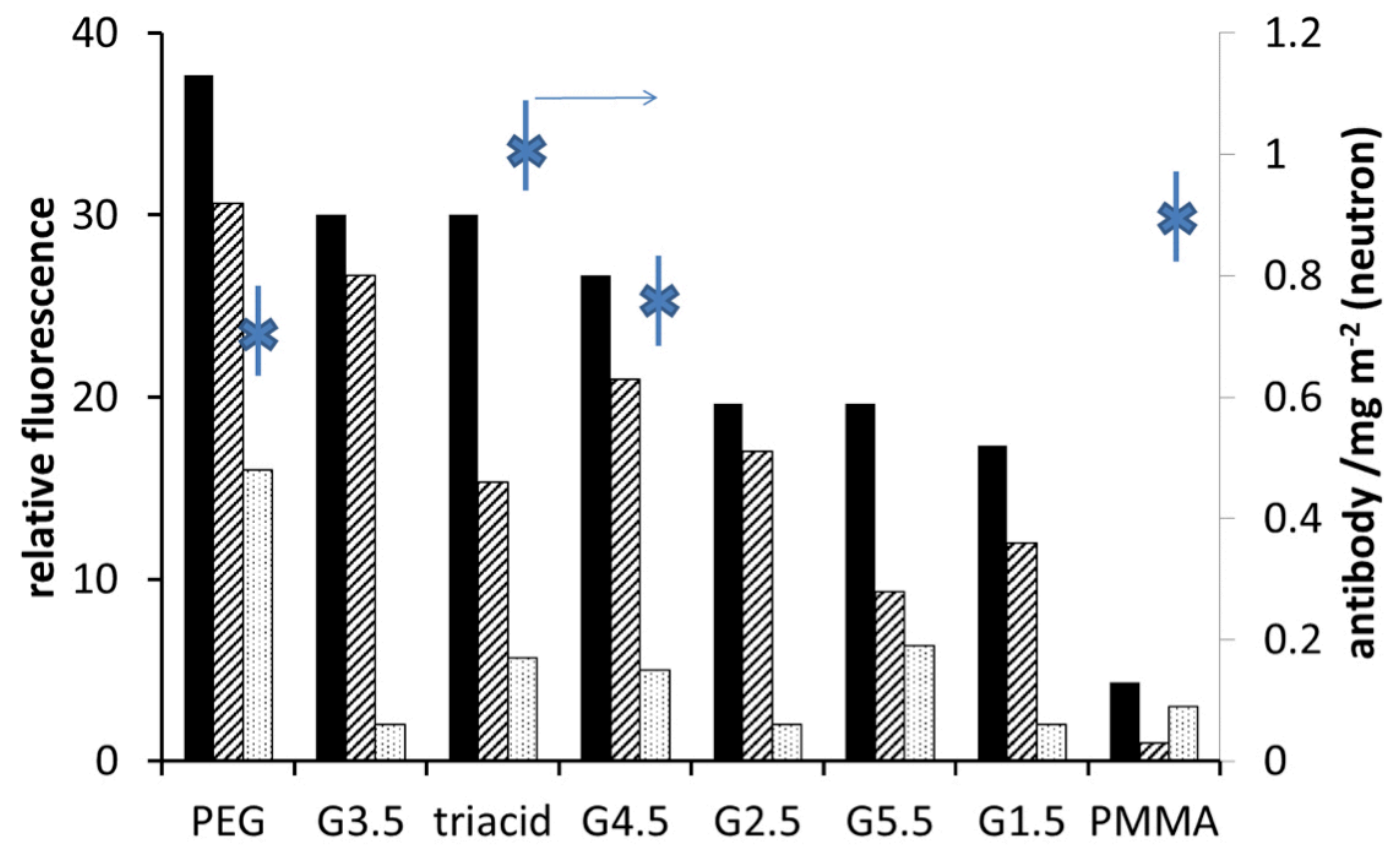

(A) surface amount of antibody $\square$ specific antigen bound non-specific antigen bound

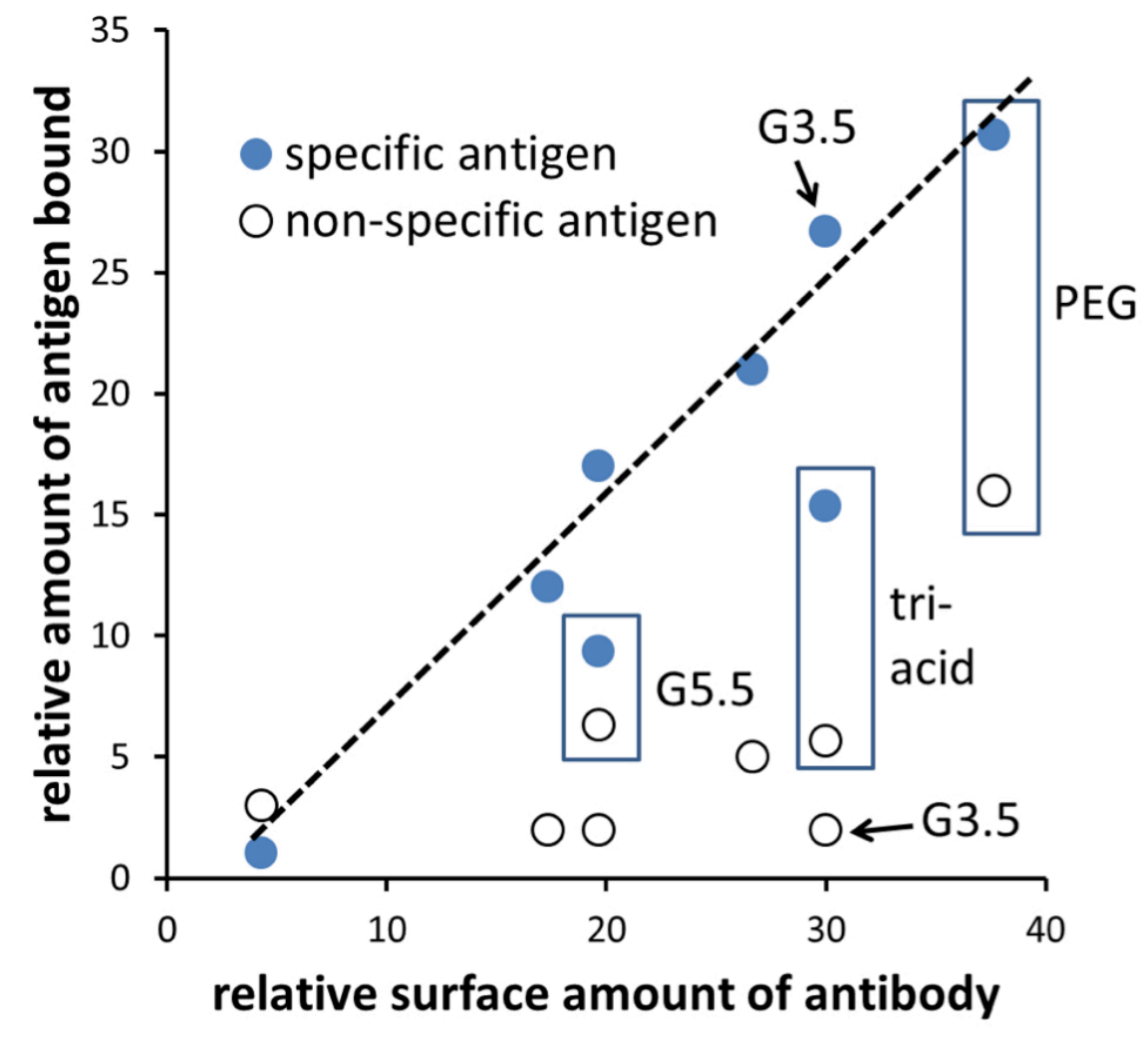

(B) 


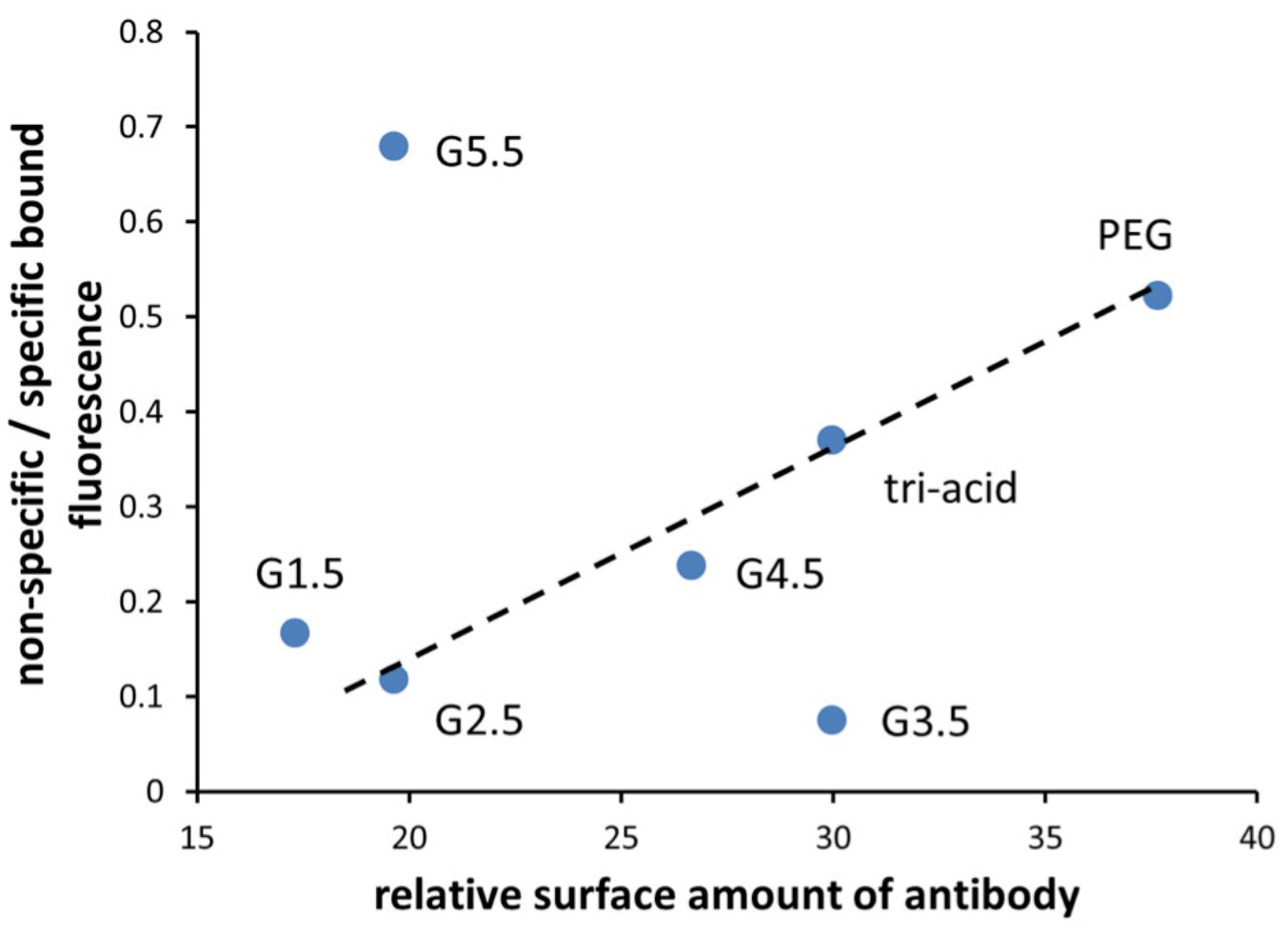

(C)

FigURE 2. (A) (LH scale) Relative fluorescence signal for the covalently-bound antibody, binding of its specific antigen and of a non-specific antigen for a range of surface linkers. (RH scale) Surface density of antibody, determined by neutron reflection. PMMA here refers to the oxidised surface. (B) Fluorescence signal for both specifically and nonspecifically bound antigen against the relative amount of surface bound antibody determined by fluorescence. (C) Fluorescence signal ratio of non-specific / specific antigen against the relative amount of surface bound antibody determined by fluorescence. Specific and non-specific antigen concentration for fluorescence measurement: $5 \mu \mathrm{g} / \mathrm{mL}$

Figure 3 shows the data for the series of dendrimer linkers. For the G3.5 linker, there is an apparent optimum in fluorescence signal from bound specific antigen associated with a low signal from bound non-specific antigen. Figure 2A shows that the surface-bound antibody fluorescence was the second-largest for this linker and figure $2 \mathrm{C}$ shows that the nonspecific to specific fluorescence ratio was the lowest of all the linkers studied. 


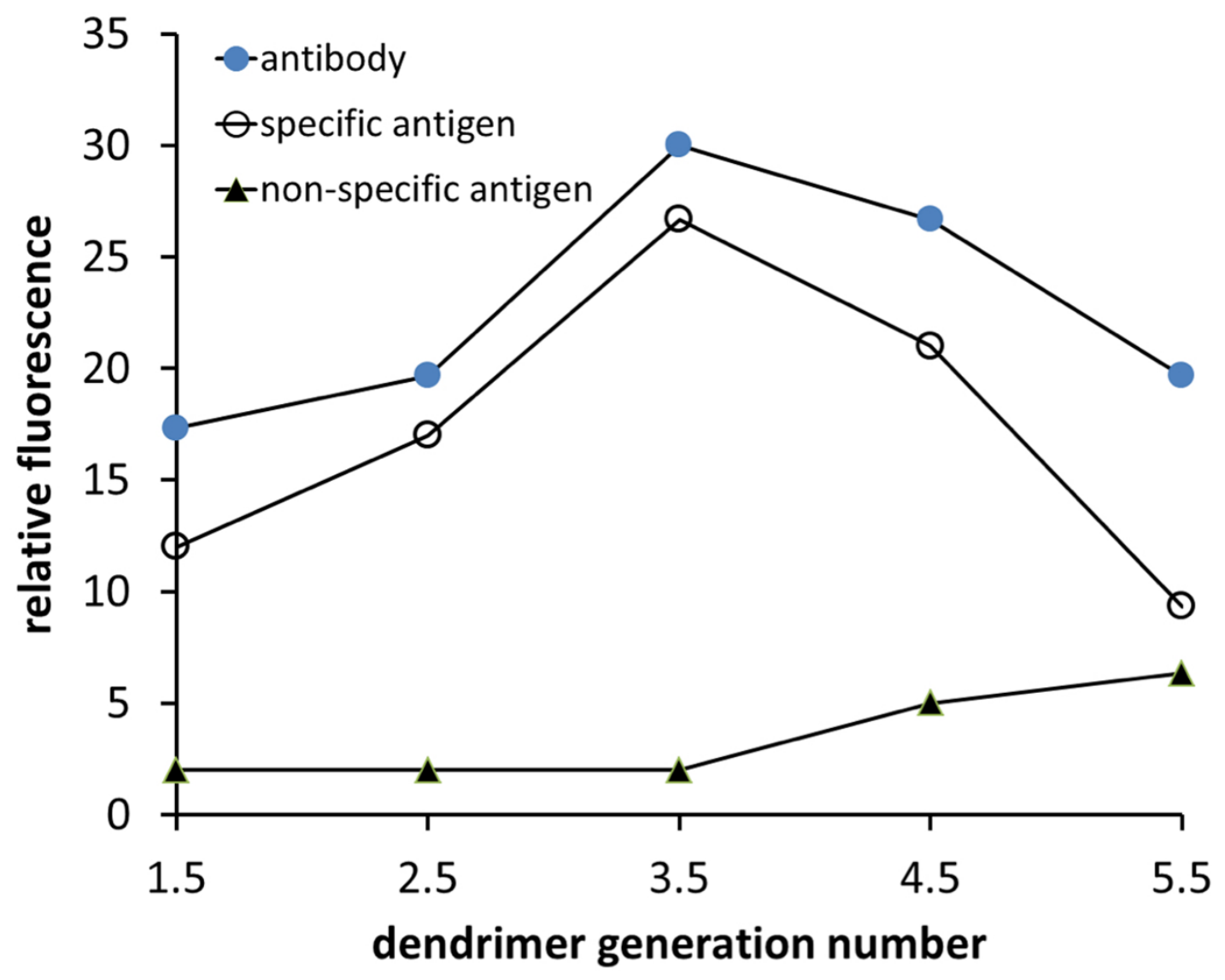

FIGURE 3. Variation of relative fluorescence signals across the series of dendrimer linkers

B. Neutron reflection shows that the effect of surface functionalization and covalent attachment is to eliminate multi-layer antibody attachment and that all covalently linked surfaces have similar surface-bound amount of antibody, but that there are some important differences in surface structure and in antigen bound per surface antibody.

Tables 1 and 2 shows surface amounts and layer thicknesses determined by neutron reflection. In all cases, the interfacial roughness was 0.4-0.7 $\mathrm{nm}$. AFM confirmed that immersion in buffer caused roughening of the surface (ESI, Figure S.1). The surface layers 
contain water (see below) and we assume that absorption of water caused the roughening. For comparison, Table 1 shows the characterization of the antibody layer formed by simple adsorption on untreated PMMA ( $\mathrm{pH}$ 7.4) followed by washing. This was a thick multilayer, containing a large proportion of water, which can be compared with the model of randomly stacked plates previously described for antibody adsorption at this $\mathrm{pH}$ onto a silica surface [32, 39-41]. There was an inner layer of thickness $4.1 \mathrm{~nm}$ consistent with a 'flat-on' configuration of adsorbed antibody. Most of the surface-bound antibody was inactive for binding of antigen.

Table 1 Surface structure and antigen binding of layer formed by adsorption of goat-anti human-IgG onto untreated PMMA.

Untreated PMMA, simple adsorption

\begin{tabular}{l|l|l|l|l|l}
\hline \multicolumn{2}{l|}{ antibody } & antigen & \multirow{2}{*}{$10^{3}$ xantibody / $\mathrm{nm}^{2}$} & Antigen / antibody \\
\cline { 1 - 3 } $\begin{array}{l}\text { Layer 1 } \\
\text { Lhickness /nm }\end{array}$ & Layer 3 & & & \\
\hline 4.1 & 18 & 11 & 6 & 7.7 & 0.05 \\
Water volume fraction & & & \\
\cline { 1 - 3 } 0.85 & 0.92 & 0.97 & 0.99 &
\end{tabular}

The covalently-attached linker layers were well-described by a single-layer model. Two layer models, with an inner dense layer and an outer hydrated layer also fit well but the slight improvement in fit did not justify the extra parameters required. Table 2 shows that the multilayer structure for the antibody layer was eliminated by surface modification to generate a carboxylated surface followed by covalent linkage of the antibody ( $\mathrm{pH}$ 7.4). This included oxidation of the PMMA without further use of a linker. The deduced linker layer thicknesses can be compared with nominal molecular dimensions, obtained from simple ChemDraw models for the triacid $(0.85 \mathrm{~nm})$ and $\mathrm{PEG}_{4}(1.8 \mathrm{~nm})$ and from the diameter of gyration of dendrimers in aqueous solution given in literature Brownian 
dynamics simulations $[42,43](3.52 \mathrm{~nm}$ for G4.5). These results are consistent with a close-packed linker layer for triacid, and with the G4.5 layer being collapsed and densified. These two linker layers also had a small volume fraction of water, consistent with this interpretation. The $\mathrm{PEG}_{4}$ layer may have been somewhat collapsed but also had a significant volume fraction of water. The layers associated with bound antibody and antigen were highly hydrated.

Table 2 Surface density and layer thickness for the various linkers and the subsequent antibody attachment and antigen binding $(100 \mu \mathrm{g} / \mathrm{mL})$.

\begin{tabular}{|c|c|c|c|c|}
\hline & $\mathrm{PEG}_{4}$ & Tri-acid & G4.5 & Oxidised PMMA \\
\hline \multicolumn{5}{|l|}{ Linker layer } \\
\hline Thickness / nm & $1.34 \pm 0.03$ & $0.91 \pm 0.01$ & $1.27 \pm 0.05$ & \\
\hline $\begin{array}{l}\text { Water volume } \\
\text { fraction }\end{array}$ & $0.16 \pm 0.01$ & $0.07 \pm 0.01$ & $0.06 \pm 0.01$ & \\
\hline
\end{tabular}

Antibody layer

\begin{tabular}{l|l|l|l|l}
\hline Thickness / nm & $4.4 \pm 0.1$ & $5.0 \pm 0.1$ & $4.0 \pm 0.1$ & $4.0 \pm 0.1$ \\
\hline $\begin{array}{l}\text { Water volume } \\
\text { fraction }\end{array}$ & $0.879 \pm 0.002$ & $0.836 \pm 0.006$ & $0.852 \pm 0.006$ & $0.830 \pm 0.010$ \\
\hline $\mathrm{mg} / \mathrm{m}^{2}$ & $0.73 \pm 0.05$ & $0.95 \pm 0.07$ & $0.82 \pm 0.06$ & $0.98 \pm 0.01$ \\
\hline
\end{tabular}

Antigen layer

\begin{tabular}{l|l|l|l|l}
\hline Thickness / $\mathrm{nm}$ & $7.2 \pm 0.3$ & $4.2 \pm 0.2$ & $3.7 \pm 0.2$ & $3.7 \pm 0.1$ \\
\hline $\begin{array}{l}\text { Water volume } \\
\text { fraction }\end{array}$ & $0.964 \pm 0.003$ & $0.948 \pm 0.006$ & $0.949 \pm 0.006$ & $0.943 \pm 0.006$ \\
\hline $\mathrm{mg} / \mathrm{m}^{2}$ & $0.36 \pm 0.04$ & $0.30 \pm 0.04$ & $0.26 \pm 0.04$ & $0.29 \pm 0.04$ \\
\hline $\begin{array}{l}\text { Mole ratio } \\
\text { antigen / antibody }\end{array}$ & 0.49 & 0.32 & 0.32 & 0.30
\end{tabular}

There were small changes in the thickness of the linker layer caused by antibody binding, though these were arguably within the errors of model fitting: the G4.5 layer expanded 
slightly, the tri-acid compressed slightly and the $\mathrm{PEG}_{4}$ was unchanged (see ESI for fitting figures, S.6-S.34). The deduced antibody layer thicknesses can be compared with antibody dimensions $(3.8 \times 8.5 \times 14.2 \mathrm{~nm})$ to indicate on all the surfaces 'flat-on' adsorption dominates, as previously described. Given that the antibody is bound as a monolayer with its long dimension approximately parallel to the surface, then the measured surface amounts of antibody indicate a surface coverage of $\sim 50 \%$. For the study of antigen binding by neutron reflection, the antigen concentration used was sufficient to saturate the surface: a lower concentration, $10 \mu \mathrm{g} / \mathrm{mL}$, gave a barely detectable layer although such a concentration gave a good signal in the much more sensitive fluorescence measurement. Given that the antigen, $\operatorname{IgG}$, is also an antibody, then the layer dimensions imply that, apart from the case with the $\mathrm{PEG}_{4}$ linker, the antigen was also bound substantially 'flat on' to the interface. The high binding ratio of antigen/antibody indicates that the bound antigen occupied a substantial fraction of the surface - if this were not the case then the neutron reflection experiment, being a surface averaging method, would not be able to detect it. The striking effect is that the large variation in adsorbed amount across the different preparations, deduced from the fluorescence measurement (Figure 2) was not shown by neutron reflection. In neutron reflection (and thus at higher surface amount) the amount of surface-bound antibody and the corresponding antigen binding varied by less than $50 \%$ across the different surfaces. The variation was much greater in the fluorescence measurement. A particular example is for the un-modified, oxidized PMMA surface, where neutron reflection showed similar surface amounts to those found on the other surfaces whereas the fluorescence measurement showed a low signal. 
The surface modified with the $\mathrm{PEG}_{4}$ linker stands out in its behavior. The calculated scattering length density profile (ESI) shows a layer that does not have a clearly recognizable boundary, in contrast to the profiles calculated for the other linkers. The water volume fraction of the layer was significantly greater than that for the other linkers. To show this result in a different way that is more clearly interpretable with a physical model, figure 4 shows the calculated volume fraction of linker within the layer, as a function of position. This diagram includes the effect of the surface roughness $(\approx 0.5 \mathrm{~nm})$. It shows that the $\mathrm{PEG}_{4}$ layer had a broader spread of volume fraction than the triacid or dendrimer layers, interpretable as a "fuzzy" layer. The amount of bound antibody was very comparable to that found for the other surfaces. The binding ratio of antigen/antibody, and the thickness increase on antigen binding, was significantly greater than that for the other surfaces. 

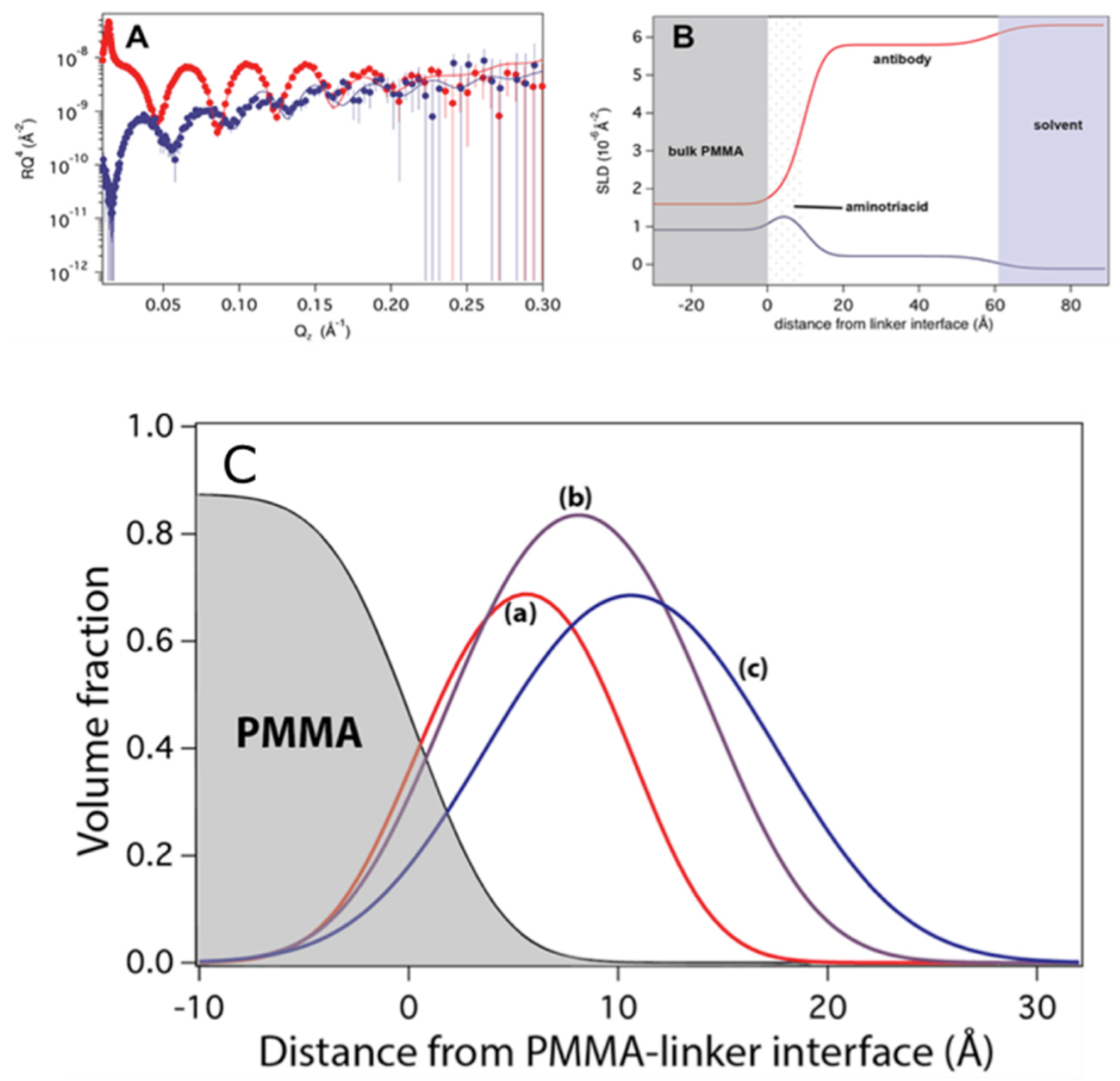

FiguRE 4. (A, B) Example of neutron reflection and derived scattering length density profile (triacid linker with coupled antibody): A -measured and fitted normalised reflection, $R\left(R Q^{4}\right.$ vs wave vector, $\left.Q\right)$; B- fitted scattering length density in $\mathrm{D}_{2} \mathrm{O}$ (red) and $\mathrm{H}_{2} \mathrm{O}$ (blue). (C) Calculated volume fraction of linker as a function of position through the linker layer, contrasting the triacid (a) and dendrimer (b) linkers with $\mathrm{PEG}_{4}$ (c). There was a small amount of absorbed water within the PMMA layer.

\section{Discussion}

For practical application, in a fluorescence immunoassay, the ratio of non-specific to specific binding, and the magnitude of the specific binding signal are of most concern. 
The objective here was to determine to what extent these signals could be influenced by appropriate choice of surface chemistry, and to deduce what factors might be driving the observed effects. The complementary information from the two measurement methods, taken with knowledge of the limitations imposed by each, leads to an interpretation of the effects of surface structure on the binding and on the signal.

Neutron reflection gives a reliable estimate of the amount of different substances present at the interface, averaged over the area interrogated, since this information requires only knowledge of the atomic composition of the materials at the interface and the neutron scattering lengths for each atomic species. Thus, the inconsistency between the amounts of surface-bound antibody, and then of the different amounts of captured antigen, indicated by the neutron and fluorescence methods (Table 1 and Figure 2) needs first to be resolved. By reference to figure 5, which illustrates schematically possible interpretations for the different surface effects seen in the two different measurements, we first discuss the variation in the fluorescence signal for surface-bound antibody. The conditions used for antibody binding were the same for both neutron and fluorescence measurements, so the amount of surface bound antibody should be the same for the samples used for the two different measurements. The neutron measurement will give the absolute surface amount, and this was very similar across all surfaces. Fluorescence measurements are susceptible to self-quenching, and differing degrees of self-quenching dependent on the surface linker used could be the explanation. A lower fluorescence signal on one surface compared to another therefore has a reasonable explanation in a self-quenching effect, illustrated in figure 5 (ii), which is very different across the different surfaces: much less for the $\mathrm{PEG}_{4}$ surface than for the oxidized PMMA. The fluorophores are attached to lysines on the 
outside of the antibody, and there are a number of these that are distributed essentially randomly over the antibody. Cyanine dye self-quenching could have a range of up to $\sim 7 \mathrm{~nm}$. If the antibody is attached in a 'flat-on' configuration with surface footprint $\sim 14 \mathrm{~nm} \times 9$ $\mathrm{nm}$ and surface coverage $\sim 0.5$ monolayer, then it is possible that small changes in surface loading and surface configuration could have a significant effect on self-quenching of the fluorophores. This however cannot simply be an effect of small variations in surface density of the antibody, since the surface with the highest antibody density, the triacid, showed one of the highest fluorescence signals. Neutron reflection, however, shows only an average surface density. If the antibody was clustered on the surface, to a degree that was different on the different surfaces, then the neutron reflection could indeed show very similar average amounts of surface-bound antibody, but the fluorescence self-quenching could be very different.

Next, figure 5 illustrates two different models for the variation across the surface of the signal due to captured antigen. The neutron measurement is of a surface that is saturated in the antigen. At the antigen concentration used for the fluorescence measurement, the neutron signal was barely detectable showing that the fluorescence measurement was of a surface that was far from saturation. Figure 5(i) illustrates that if the binding constant of antigen to surface-bound antibody was different for the different surface preparations, then the fluorescence measurement could reflect different surface amounts at low coverage. Figure 5(ii) illustrates the hypothesis that the effect could arise if there was self-quenching of the fluorescence signal due to captured antigen that was different on the different surfaces. Surface clustering of the antibodies, different across the different surfaces, provides an explanation that is consistent with both mechanisms 
illustrated in figure 5. An effect on surface binding constant for antigen could arise due to different steric hindrance to binding on the different surfaces. Different steric hindrance on the different surfaces could arise if the bound antibodies are clustered, differently on the different surfaces. Surface clustering of the bound antibody would also evidently lead to surface clustering of the captured antigen, and this could then cause surface self-quenching of the captured antigen fluorescence signal. Figure 2B shows that, over the different surfaces, the fluorescence signal due to captured antigen varies linearly with the fluorescence signal for surface antibody, with two outliers: the triacid and the G5.5 dendrimer. Since the fluorescence signal due to surface antibody is most likely influenced by surface self-quenching, the result in figure 2B implies that the same mechanism, surface self-quenching, is behind the variation of signal due to captured antigen. The outliers may reflect an effect of variation of surface binding constant.

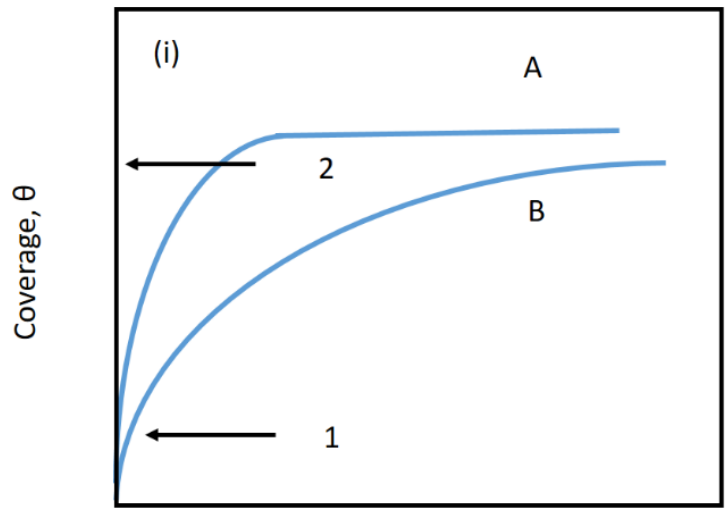

Solution concentration

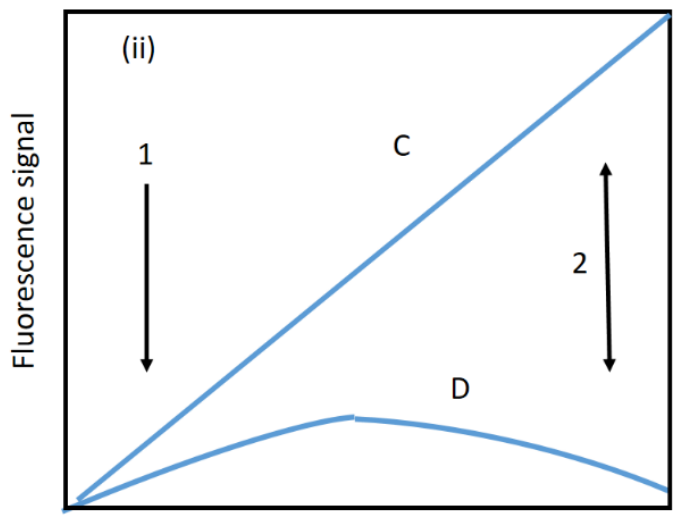

Coverage, $\theta$

FIGURE 5. Illustration of mechanisms for differing results from fluorescence and neutron reflection measurements. (i) Effect of different surface binding constant for antigen, on two different surfaces labelled A and B. Arrow 1 indicated the surface coverage range probed by fluorescence whilst 2 indicates that probed by neutron reflection. The two surfaces would show different results for the two measurements. (ii) Effect of surface selfquenching on the fluorescence signal: $\mathrm{C}$ illustrates the signal for an ideal surface without 
self-quenching whereas D illustrates that for a surface with significant self-quenching, increasing with increasing surface coverage. Arrow 1 indicates the coverage probed by fluorescence measurement of antigen binding. Arrow 2 indicates the coverage probed by both fluorescence and neutron reflection measurement of antibody loading.

Can the results suggest a mechanism by which surface clustering of the antibody could depend on the nature of the linker used? A mechanism by which such clustering might be dependent on the nature of the linker is indeed suggested, by the comparison of antibody loading on the untreated and bare, oxidized PMMA, determined by neutron reflection. On the untreated PMMA, the antibody adsorbed in a thick multilayer. The reaction on the oxidized PMMA, which would occur following an initial physical adsorption, resulted in a monolayer of antibody on the surface. The oxidized PMMA is carboxylated and thus charged; the untreated PMMA is not. Hence the key to interpretation could be in the charge on the surface in relation to the charge on the antibody. A combination of local electrostatic interactions between antibody molecules, and between antibody and surface could lead to clustering of the surface-bound antibodies dependent on the surface charge density. Such clustering has previously been inferred for surface adsorption of an antibody [44]. The surface clusters could then be locked in place by the coupling reaction to the underlying linker. The rate of the coupling reaction in relation to the rate of formation of surface clusters in the initial, physical adsorption step, might then be an important factor. This leads to a second idea for a control parameter: the rate of the surface coupling reaction could to a degree depend on conformational flexibility of the linker and its physical interaction with the antibody. The most conformationally flexible linker layer was the $\mathrm{PEG}_{4}$, as indicated by the "fuzziness" revealed by neutron reflection. This was the surface with the largest antibody fluorescence. It is reasonable to assume that the least conformationally flexible surface was the oxidized PMMA, which was the surface 
with the smallest antibody fluorescence. The comparisons thus indicate surface charge, and conformational flexibility of the linker as controlling factors driving antibody clustering on the surface resulting from the coupling reaction.

Thus, we interpret the results as showing that, to maximize the fluorescence signal, the surface should be configured to minimize self-quenching of the fluorophore. Selfquenching caused presumably by clustering of the antibody on the surface carries though into self-quenching of the signal due to bound antigen. We have indeed shown elsewhere the effects of surface self-quenching on the signal generation in a fluorescence immunoassay [45].

The idea of conformational flexibility that we introduced above as a factor which could act against clustering of antibody during the surface coupling is also useful in considering the maximum fluorescence signal that could be achieved. Again, this idea comes from comparison of the results of fluorescence and neutron reflection. The surface that showed the highest fluorescence signal generation was that with the $\mathrm{PEG}_{4}$ linker. Neutron reflection showed that this surface had the highest antigen/antibody binding ratio: the saturation coverage of captured antigen on this surface was the greatest of all. In other work, we showed that steric hindrance of access of the antigen to the antibody binding site was the major determinant of antigen/antibody binding ratio [39, 41]. We attribute the high binding ratio to the conformational flexibility of the $\mathrm{PEG}_{4}$ linker counteracting the effects of steric hindrance.

The ratio of non-specific to specific binding signal is important to minimize in practical immunoassay. We can develop some tentative conclusions based on the general trend shown in Figure 2C. The signal ratio increases with the fluorescence signal for 
surface-bound antibody with two notable outliers, both from the dendrimer series. The signal ratio was by far the worst for the oxidized PMMA surface, for which we deduced antibody clustering. Of the other surfaces, apart from the G5.5 dendrimer, the signal ratio was worst for the $\mathrm{PEG}_{4}$ linker, which had the highest antigen/antibody binding ratio determined by neutron reflection, and the highest fluorescence signal reflecting the least amount of surface clustering and self-quenching. As the deduced surface clustering of antibody increases, reflected by the decrease in the antibody fluorescence signal, the nonspecific / specific fluorescence ratio decreases. We suggest that the major cause of nonspecific binding was the antibody itself, specifically the non-binding regions. If the antibody were clustered, its effect on non-specific binding would presumably decrease. This deduction suggests the possibility of engineering the non-binding regions of antibodies in order to minimize non-specific binding on surfaces that have been optimized to maximize the active antibody loading for specific binding. If antibody clustering is very significant, then non-specific binding to the part of the surface that is not covered with antibody would need to be considered, and would depend on the nature of the linker. We discuss later that this effect might account for the G5.5 outlier on figure 2C.

The fluorescence data for the dendrimer series suggest interesting possibilities for maximizing specific signal whilst minimizing non-specific signal. For the dendrimer series, we observe that the non-specific fluorescence signal was low and constant for G1.5 to 3.5 then rose for G4.5 and 5.5. The specific signal rose for G1.5 to 3.5 then fell for G4.5 and 5.5. The non-specific to specific binding signal ratio was worst for G5.5 because the specific binding signal had fallen but the non-specific signal had risen. It was best for G3.5 
because the specific binding signal was maximal but the non-specific binding signal had not risen.

Computational studies show that dendrimers can undergo a temperature-driven expansion- collapse transition on a surface, dependent on the linker length and dendrimer generation number [46]. At a fixed temperature below the transition temperature, the fraction of adsorbed monomers of the dendrimer, reflecting the degree of flattening of the dendrimer onto the surface, decreases as the generation number increases. The neutron reflection result for the G4.5 dendrimer shows that this molecule was partly (but not completely) collapsed, implying that the temperature was above that for the collapse transition. Since the transition temperature decreases as generation number increases[46] the implication is that the G1.5 dendrimer would be more collapsed, and that the layer would progressively expand as the generation number increased.

The following ideas seem consistent with the data. The G1.5 dendrimer is almost completely collapsed and so represents a surface with a density of carboxylate groups on which to link antibody lower than that for the tri-acid and $\mathrm{PEG}_{4}$ linkers, and without the conformational flexibility of the $\mathrm{PEG}_{4}$ linker. With increase of generation number, the surface carboxylate density increases so the amount of bound antibody should increase. The increase in amount of bound antibody is reflected in the increase in fluorescence signal both for the antibody and for the captured antigen. However, with further increase of generation number, the fluorescence signal due to the antibody falls. Self-quenching due to clustering of the antibody was deduced above as the mechanism. This clustering may be driven by the increasing charge on the interface as the surface carboxylate density increases. In the extreme the surface might approach the behavior of the oxidized PMMA. 
Clustering of the antibody leads to both steric effects on antigen capture and self-quenching of the captured antigen fluorescence: both effects would decrease the specific signal. Nonspecific binding occurring on the part of the surface not covered with antibody would account for the increase in non-specific signal. The balance between all the effects discussed here as controlling the fluorescence signals - surface charge, antibody density, surface self-quenching, conformational flexibility - seem to have operated optimally for the G3.5 surface. These deductions at first sight seem at odds with earlier results concerning the use of dendrimers as linkers on nanoparticles, where the binding capacity increased steadily with generation number [35]. However, in that case, attachment onto the strongly curved surface of a nanoparticle would have mitigated the steric interaction and clustering effects that we propose here for a flat surface.

\section{IV.SUMMARY AND CONCLUSIONS}

When surface-bound antibodies are used in a fluorescence immunoassay, the sensitivity can be strongly affected by antibody clustering on the surface and surface selfquenching. Self-quenching and clustering can be mitigated by use of a binding surface that has conformational flexibility. Conformational flexibility also promotes a high antigen to antibody binding ratio and hence a high signal. The strongest signal was achieved with a $\mathrm{PEG}_{4}$ linker, which caused a conformationally flexible interface. Non-specific adsorption was promoted by the antibodies themselves, suggesting the possibility of engineering the non-binding regions to minimize this. Non-specific adsorption onto areas of the surface not carrying antibody was also promoted by antibody clustering. With increase of dendrimer generation number, surfaces with dendrimers as linkers appeared to progress from a collapsed dendrimer layer through layers which were less collapsed. A G3.5 
dendrimer as linker offered the best combination of high fluorescence signal intensity and low non-specific to specific fluorescence signal. The effect appeared to be a balance between the effects of antibody loading and antibody clustering. Only by a combination of techniques with complementary attributes for measurement of surface properties, here fluorescence and neutron reflection applied in-situ, was it possible to resolve the different influences on linker thickness, surface activity for antibody binding, antigen to antibody binding ratio and surface conformational change.

\section{ASSOCIATED CONTENT}

Electronic Supporting Information (ESI). A detailed procedure and data on the Atomic Force Microscopy images, Water contact Angles of the surfaces, Fluorescence images and Neutron Reflection data is available free of charge.

1. Brault ND, Gao C, Xue H, Piliarik M, Homola J, Jiang S, Yu Q (2010) Ultra-low fouling and functionalizable zwitterionic coatings grafted onto $\mathrm{SiO}_{2}$ via a biomimetic adhesive group for sensing and detection in complex media. Biosens. Bioelectron. 25(10): 22762282

2. Wang F, Anderson M, Bernards MT, Hunt HK (2015) PEG Functionalization of Whispering Gallery Mode Optical Microresonator Biosensors to Minimize Non-Specific Adsorption during Targeted, Label-Free Sensing. Sens. 15(8): 18040-18060

3. Kim MI, Kim MS, Woo M-A, Ye Y, Kang KS, Lee J, Park HG (2014) Highly efficient colorimetric detection of target cancer cells utilizing superior catalytic activity of graphene oxide-magnetic-platinum nanohybrids. Nanoscale 6(3): 1529-1536

4. Jeong J-W, Kim MK, Cheng H, Yeo W-H, Huang X, Liu Y, Zhang Y, Huang Y, Rogers JA (2014) Capacitive Epidermal Electronics for Electrically Safe, Long-Term Electrophysiological Measurements. Adv. Healthcare Mater. 3(5): 642-648

5. Reimhult E, Hook F (2015) Design of Surface Modifications for Nanoscale Sensor Applications. Sens. 15(1): 1635-1675

6. Yu Q, Wang Q, Li B, Lin Q, Duan Y (2014) Technological Development of Antibody Immobilization for Optical Immunoassays: Progress and Prospects. Crit. Rev. Anal. Chem. 45(1): $62-75$ 
7. Rusmini F, Zhong ZY, Feijen J (2007) Protein immobilization strategies for protein biochips. Biomacromol. 8(6): 1775-1789

8. Wong LS, Khan F, Micklefield J (2009) Selective Covalent Protein Immobilization: Strategies and Applications. Chem. Rev. 109(9): 4025-4053

9. Jonkheijm P, Weinrich D, Schroder H, Niemeyer CM, Waldmann H (2008) Chemical strategies for generating protein biochips. Angew. Chem. Int. Ed. 47(50): 9618-9647

10. Kohn M (2009) Immobilization strategies for small molecule, peptide and protein microarrays. J. Pept. Sci. 15(6): 393-397

11. Yeo DSY, Panicker RC, Tan LP, Yao SQ (2004) Strategies for immobilization of biomolecules in a microarray. Comb. Chem. High Throughput Screen 7(3): 213-221

12. Fu JL, Reinhold J, Woodbury NW (2011) Peptide-Modified Surfaces for Enzyme Immobilization. PloS One 6(4): e18692

13. Kosobrodova E, Jones RT, Kondyurin A, Chrzanowski W, Pigram PJ, McKenzie DR, Bilek MM (2015) Orientation and conformation of anti-CD34 antibody immobilised on untreated and plasma treated polycarbonate. Acta Biomater. 19: 128-137

14. Vashist SK, Lam E, Hrapovic S, Male KB, Luong JHT (2014) Immobilization of Antibodies and Enzymes on 3-Aminopropyltriethoxysilane-Functionalized Bioanalytical Platforms for Biosensors and Diagnostics. Chem. Rev. 114(21): 11083-11130

15. Crivianu-Gaita V, Thompson M (2015) Immobilization of Fab' fragments onto substrate surfaces: A survey of methods and applications. Biosens. Bioelectron. 70: 167-180

16. Alves NJ, Kiziltepe T, Bilgicer B (2012) Oriented surface immobilization of antibodies at the conserved nucleotide binding site for enhanced antigen detection. Langmuir 28(25): 9640-9648

17. Hui JZ, Al Zaki A, Cheng ZL, Popik V, Zhang HT, Prak ETL, Tsourkas A (2014) Facile Method for the Site-Specific, Covalent Attachment of Full-Length IgG onto Nanoparticles. Small 10(16): 3354-3363

18. Ericsson EM, Enander K, Bui L, Lundstrom I, Konradsson P, Liedberg B (2013) SiteSpecific and Covalent Attachment of His-Tagged Proteins by Chelation Assisted Photoimmobilization: A Strategy for Microarraying of Protein Ligands. Langmuir 29(37): 11687-11694

19. Yang HM, Bao RM, Cheng YZ, Tang JB (2015) Site-specific covalent attachment of an engineered Z-domain onto a solid matrix: An efficient platform for 3D IgG immobilization. Anal. Chim. Acta 872: 1-6 
20. Wold ED, McBride R, Axup JY, Kazane SA, Smider VV (2015) Antibody Microarrays Utilizing Site-Specific Antibody - Oligonucleotide Conjugates. Bioconjugate Chem. 26(5): $807-811$

21. Liu F, Wang L, Wang HW, Yuan L, Li JW, Brash JL, Chen H (2015) Modulating the Activity of Protein Conjugated to Gold Nanoparticles by Site-Directed Orientation and Surface Density of Bound Protein. ACS Appl. Mater. Interfaces 7(6): 3717-3724

22. Wang C, Feng B (2015) Research progress on site-oriented and three-dimensional immobilization of protein. Mol. Biol. 49(1): 1-20

23. Camarero JA (2008) Recent developments in the site-specific immobilization of proteins onto solid supports. Biopolymers 90(3): 450-458

24. Sung D, Park S, Jon S (2012) Facile immobilization of biomolecules onto various surfaces using epoxide-containing antibiofouling polymers. Langmuir 28(9): 4507-4514

25. Weidner T, Apte JS, Gamble LJ, Castner DG (2010) Probing the Orientation and Conformation of $\alpha$-Helix and $\beta$-Strand Model Peptides on Self-Assembled Monolayers Using Sum Frequency Generation and NEXAFS Spectroscopy. Langmuir 26(5): 34333440

26. Baio JE, Weidner T, Baugh L, Gamble LJ, Stayton PS, Castner DG (2012) Probing the Orientation of Electrostatically Immobilized Protein G B1 by Time-of-Flight Secondary Ion Spectrometry, Sum Frequency Generation, and Near-Edge X-ray Adsorption Fine Structure Spectroscopy. Langmuir 28(4): 2107-2112

27. Fears KP, Clark TD, Petrovykh DY (2013) Residue-Dependent Adsorption of Model Oligopeptides on Gold. J. Am. Chem. Soc. 135(40): 15040-15052

28. Bilek MM, McKenzie DR (2010) Plasma modified surfaces for covalent immobilization of functional biomolecules in the absence of chemical linkers: towards better biosensors and a new generation of medical implants. Biophys. Rev. 2(2): 55-65

29. Fears KP, Petrovykh DY, Photiadis SJ, Clark TD (2013) Circular Dichroism Analysis of Cyclic $\beta$-Helical Peptides Adsorbed on Planar Fused Quartz. Langmuir 29(32): 1009510101

30. Moore CJ, Monton H, O'Kennedy R, Williams DE, Nogues C, Crean C, Gubala V (2015) Controlling colloidal stability of silica nanoparticles during bioconjugation reactions with proteins and improving their longer-term stability, handling and storage. J. Mater. Chem. B 3(10): 2043-2055

31. Wiseman ME, Frank CW (2012) Antibody Adsorption and Orientation on Hydrophobic Surfaces. Langmuir 28(3): 1765-1774 
32. Xu H, Zhao XB, Lu JR, Williams DE (2007) Relationship between the structural conformation of monoclonal antibody layers and antigen binding capacity. Biomacromolecules 8(8): 2422-2428

33. Ali U, Karim KJBA, Buang NA (2015) A Review of the Properties and Applications of Poly (Methyl Methacrylate) (PMMA). Polym. Rev. 55(4): 678-705

34. Le NCH, Gubala V, Clancy E, Barry T, Smith TJ, Williams DE (2012) Ultrathin and smooth poly(methyl methacrylate) (PMMA) films for label-free biomolecule detection with total internal reflection ellipsometry (TIRE). Biosens. Bielectron. 36(1): 250-256

35. Gubala V, Crean C, Nooney R, Hearty S, McDonnell B, Heydon K, O'Kennedy R, MacCraith BD, Williams DE (2011) Kinetics of immunoassays with particles as labels: effect of antibody coupling using dendrimers as linkers. Analyst 136(12): 2533-2541

36. James M, Nelson A, Holt SA, Saerbeck T, Hamilton WA, Klose F (2011) The multipurpose time-of-flight neutron reflectometer "Platypus" at Australia's OPAL reactor. Nucl. Instrum. Methods Phys. Res., Sect. A 632(1): 112-123

37. Nelson A (2006) Co-refinement of multiple-contrast neutron/X-ray reflectivity data using MOTOFIT. J. Appl. Crystallogr. 39: 273-276

38. Heinrich F, Ng T, Vanderah DJ, Shekhar P, Mihailescu M, Nanda H, Losche M (2009) A New Lipid Anchor for Sparsely Tethered Bilayer Lipid Membranes. Langmuir 25(7): 4219-4229

39. $\mathrm{Xu} \mathrm{H}, \mathrm{Lu} \mathrm{JR}$, Williams DE (2006) Effect of surface packing density of interfacially adsorbed monoclonal antibody on the binding of hormonal antigen human chorionic gonadotrophin. J. Phys. Chem. B 110(4): 1907-1914

40. Xu H, Williams DE, Lu JR (2005) The effect of antibody surface packing density on its antigen binding capacity. Prog. Nat. Sci. 15: 139-144

41. Xu H, Zhao XB, Grant C, Lu JR, Williams DE, Penfold J (2006) Orientation of a monoclonal antibody adsorbed at the solid/solution interface: A combined study using atomic force microscopy and neutron reflectivity. Langmuir 22(14): 6313-6320

42. Maiti PK, Cagin T, Wang GF, Goddard WA (2004) Structure of PAMAM dendrimers: Generations 1 through 11. Macromolecules 37(16): 6236-6254

43. Paulo PMR, Lopes JNC, Costa SMB (2007) Molecular Dynamics Simulations of Charged Dendrimers: Low-to-Intermediate Half-Generation PAMAMs. J. Phys. Chem. B 111(36): 10651-10664

44. Lea AS, Pungor A, Hlady V, Andrade JD, Herron JN, Voss EW (1992) Manipulation of proteins on mica by atomic force microscopy. Langmuir 8(1): 68-73 
45. Vareiro M, Tranchant I, Maplin S, Zak K, Gani MM, Slevin CJ, Hailes HC, Tabor AB, Cameron PJ, Jenkins ATA, Williams DE (2008) Surface plasmon resonance-enhanced fluorescence implementation of a single-step competition assay: Demonstration of fatty acid measurement using an anti-fatty acid monoclonal antibody and a Cy5-labeled fatty acid. Anal. Biochem. 377(2): 243-250

46. Sommer JU, Klos JS, Mironova ON (2013) Adsorption of branched and dendritic polymers onto flat surfaces: A Monte Carlo study. J. Chem. Phys. 139(24): 244903 